\title{
Determinantes estructurales de las redes sociales en los hombres y las mujeres
}

\author{
Félix Requena Santos \\ Universidad de Málaga. Facul aad de Ciencias Económicas. Departamento de Sociologta \\ Campus de El Ejido. 2907 I Málaga. Spain
}

\section{Resumen}

Las redes personales de los hombres y de las mujeres, en muchos casos, difieren en composición y en tamaño. Las redes de los hombres se centran en las no familiares, mientras que fas de las mujeres se componen en su mayoría por farniliares.

Usando datos de una encuesta realizada en 1989 por el Departamento de Sociología de la Universidad de Málaga, se comprueba que las diferencias entre la composición de las redes personales de los hombres y las de las mujeres se deben fundamentalmente a diferencias estructurales respecto a los sexos. Sin embargo, las diferencias desaparecen cuando se posiciona a las mujeres en lugares sociates $y$, sobre todo, laborales similares a los de los hombres. Cuando las oportunidades son semejantes para ambos sexos, entonces las mujeres muestran redes incluso mayores y de composición más variada que los hombres.

Palabras clave: redes sociales, estructura social, género.

\section{Abstract. Structural conditionnings in men's and women's social networks}

Men's and women's personal networks often differ in composicion and range. Women's networks are more focused on family, and men's on nonkin, especially coworkers.

Using dara from a 1989 social survey undertaken by Socjology Dept. (Malaga Universiry), it find that these gender differences arise from dissimilar social structural locations of genders. Most differences disappear when women and men hold same social and labour positions. When opportunities between gender are very much alike then women show larger number and diversity of nonkin ties in their personal networks than men.

Key words: social nerworks, social structure, gender.

\section{Sumario}

Introducción Conclusiones

Datos y variables Bibliografía 


\section{Introducción}

Numerosos estudios sobre redes personales han puesto de manifiesto que los hombres y las mujeres tienen redes de un tamaño parecido (Fischer, 1982; Marsden, 1987). En el caso de la amistad, hay poca diferencia en el promedio de amigos de los hombres y de las mujeres (Booth, 1972). Sin embargo, existe una diferencia relativa en las composiciones internas de dichas redes. Tales diferencias están originadas por causas diversas como, por ejemplo, la disponibilidad del espacio social que cada uno de los sexos tiene para desarrollar los vínculos que nutren a tales redes. Así mismo, cuando se consideran las posiciones de clase social, cobra especial realce la importancia del contexto como medio para fomentar las interacciones (Deem, 1982). Como han señalado algunos investigadores, las redes de los hombres adultos son más densas que las de las mujeres adultas (Granoverter, 1974; Requena, 1991). Lo mismo se puede decir acerca de los tipos de vínculos que existen en las redes de uno y otro género: cuando se compara hombres con mujeres, aquellos tienen menos relaciones con parientes y más con compañeros de trabajo (Fischer y Oliker, 1983; Moore, 1990). Todo ello apunta a que las mujeres tienen menos oportunidad de usar las redes sociales como un recurso instrumental, del mismo modo que los hombres se benefician en mayor proporción de las ventajas que dichas redes suponen, por ejemplo, en el caso de la búsqueda de empleo o en la promoción profesional.

Otros trabajos (Miller, 1976; Fischer y Oliker, 1983) han puesto de manifiesto que las diferencias entre los géneros con respecto a las redes se atribuyen a la disposición que muestran los diferentes sexos hacia las relaciones personales, de forma que las mujeres se inclinan a mantener lazos más íntimos hacia los parientes y menos hacia los que se encuentran fuera de la familia (Winstead, 1986). Por ello es interesante matizar qué composición reticular se produce con mayor frecuencia en cada uno de los sexos.

En este estudio investigaremos los determinantes estructurales de las redes de los hombres y de las mujeres para identificar los factores de la estructura social y determinar si las diferencias de género en las redes personales pueden ser explicadas por dichos factores.

Se ha demostrado que las relaciones sociales dependen de las oportunidades de contacto social que posee el sujeto relacionado (Blau, 1977). Por sí mismo, este axioma refleja prácticamente toda la perspectiva estructural. Es decir, las relaciones ocurren dentro del marco de la oportunidad que el contexto proporciona para que se produzcan las interacciones, que más tarde se convertirán en relaciones o incluso en vínculos más duraderos. Puesto que no hay evidencia que nos haga dudar de tales hechos, nos apoyaremos en la proposición de que los hombres y las mujeres ocupan roles diferentes en la estructura social (laborales, familiares, parentesco), lo que proporciona diferentes situaciones contextuales que generan mayores oportunidades $o$, por el contrario, limitaciones para construir determinados tipos de vínculos. 
De este enunciado se pueden obtener, a su vez, una serie de proposiciones que trataremos de verificar empíricamente:

1. Ante todo, la hipótesis general de trabajo, y de la que se derivan otras, es la que trata de dar una explicación estructural de las diferencias de las redes personales entre los diferentes géneros. Las diferentes posiciones que habitualmente ocupan los hombres y las mujeres en el sistema social, del mismo modo que la desigualdad de oportunidades que tienen los diferentes sexos para acceder a recursos escasos, ya sean éstos económicos, políticos o simbólicos, hace que la composición estructural de las tedes personales de los hombres y de las mujeres sea diferente.

2. La desigualdad de oportunidades en el mercado de trabajo facilita mejores empleos (en posibilidad de promoción y de ingresos) a los hombres que a las mujeres, con lo que desde el punto de vista laboral los hombres tienen más oportunidades de relación con individuos que ocupan la posición de compañeros de trabajo. Por otra parte, el trabajo en el hogar y el cuidado de los hijos todavía impone mayores limitaciones a las mujeres que a los hombres, lo que supone mayor proporción de contacto con familiares y vecinos que pueden facilitar una ayuda eficaz en dichos trabajos dentro del hogar. De esta forma la estructura familiar también afectará a la composición de las redes como de hecho han demostrado varios investigadores para el caso de los EE. UU. (Fischer, 1982; Wellman, 1985). Sin embargo, la familia y los niños también pueden representar una oportunidad de formar más lazos con parientes y vecinos.

3. También existen otras variables estructurales relacionadas con el nivel laboral y el volumen de ingresos o con el nivel de instrucción, que a priori pueden considerarse correlacionadas positivamente con vínculos establecidos con individuos ajenos al entorno familiar.

\section{Datos y variables}

Los datos de este estudio se han obtenido de una encuesta llevada a cabo en febrero de 1989 por el Departamento de Sociología de la Universidad de Málaga. La muestra fue aleatoria y tenía un tamaño de $\mathrm{N}=609$, individuos de ambos sexos con edades comprendidas entre los 16 y 65 años que pertenecían a la población activa o bien estaban jubilados y eran residentes en la ciudad de Málaga. El nivel de confianza fue del $95,5 \%(2 \sigma)$ y el error estimado para el caso más desfavorable fue de $\pm 4,05 \%$.

El cuestionario se articuló, entre otras partes, en los siguientes bloques:

1. Características sociodemográftcas.

2. Preguntas sobre la composición y el tamaño de las redes.

3. Relaciones usadas en la búsqueda de empleo.

4. Mercado de trabajo.

5. Ocio. 
En las características sociodemográficas, las frecuencias de dicha encuesta fueron similares a las que proporciona la Encuesta de Población Activa para la misma fecha.

Aunque la estructura de las redes tiene varios componentes, el punto de mira de este estudio se centra en el rango de la red y los tipos de vínculos existentes dentro de ella; en cambio no nos centraremos en otros rasgos estructurales como la densidad o los niveles de apiñamiento. Tampoco se matiza sobre la intensidad de los lazos, fuertes o débiles (Granovetter, 1973), pues to que nos interesa es que se aprecien con mayor claridad las diferencias entre los sexos, hecho que se aprecia mejor simplificando dichas características estructurales. Hay varias medidas del rango de una red. Aquí usaremos una de las propuestas por Burt (1983), que consiste en el volument de contactos y la diversidad de los mismos; así pues, mediremos el volumen de contactos como la cantidad total de personas nombradas por cada sujeto entrevistado, y la diversidad por el número de personas nombradas para cada vínculo preguntado: familia, amigos, vecinos y compañeros de trabajo, así como la proporción de cada tipo de vínculo existente en la red personal de un sujeto.

Las variables estructurales consideradas en este trabajo son de tres tipos:

I. Sociodemográficas: sexo, edad y nivel educativo.

2. Laborales: siruación laboral (empleado o desempleado), ocupación y nivel de ingresos.

3. Situación familiar: si se encuentra casado y si hay hijos pequeños.

\section{Análisis}

Respecto al uso instrumental de las redes sociales según los diferentes sexos, usaremos como ejemplo el caso de la búsqueda de empleo'. Como se puede apreciar en la tabla 12 , los hombres como primera opción son más proclives a buscar empleo a través de sus redes personales (familia y amigos), mientras que las mujeres usan más los mecanismos formales o institucionales (anuncios en la prensa, oficina de empleo) para comenzar a buscar empleo. Sólo como último recurso las mujeres utilizan sus redes personales y principalmente los amigos más que la familia, mientras que los hombres como último recurso prestan atención a los caminos institucionalizados como es el caso de la oficina de empleo. El resultado final de la búsqueda es también diferente para los hombres y las mujeres (tabla 1b). A juzgar por los datos, aunque los hombres, como se ha dicho, ponen una intención más instrumental en el uso de sus redes personales, sin embargo éstas resultan más eficaces para las mujeres, que aun sin buscar con tanto interés a través de sus redes personales, los empleos que encuentran los obtienen con más frecuencia mediante dichas redes.

1. Un análisis más detallado y completo del uso de las redes sociales en los procesos de búsqueda $y$ acceso al empleo se puede ver en F. Requena (1991). 
Tabla 1a. Forma de buscar empleo, \% por sexos.

\begin{tabular}{|c|c|c|c|c|}
\hline En primera opción & & Hombres & Mujeres & Total \\
\hline $\begin{array}{l}\text { A través de la familia } \\
\text { A través de amigos } \\
\text { Anuncios en la prensa } \\
\text { Otros anuncios } \\
\text { INEM } \\
\text { Oposiciones } \\
\text { Yendo personalmente }\end{array}$ & $\begin{array}{l}\text { Total } \\
N\end{array}$ & $\begin{array}{c}30,6 \\
37,1 \\
3,2 \\
- \\
22,6 \\
- \\
6,5 \\
100 \\
(62)\end{array}$ & $\begin{array}{c}18,9 \\
21,6 \\
13,5 \\
- \\
35,1 \\
2,7 \\
8,1 \\
100 \\
(37)\end{array}$ & $\begin{array}{c}26,3 \\
31,3 \\
7,1 \\
- \\
27,3 \\
1 \\
7,1 \\
100 \\
(99)\end{array}$ \\
\hline
\end{tabular}

Chi-cuadrado $=9,5585$

D.F. $=5$

$\mathrm{p}=0,0888$

En última opción

A través de la familia

A través de amigos

Anuncios en la prensa

Otros anuncios

INEM

Oposiciones

Yendo personalmente

\begin{tabular}{lccc} 
& Hombres & Mujeres & Total \\
\hline & 17,2 & 7,7 & 14,3 \\
& 6,9 & 15,4 & 9,5 \\
& 20,7 & 38,5 & 26,2 \\
& - & 7,7 & 2,4 \\
& 41,4 & 23,1 & 35,7 \\
& 3,4 & - & 2,4 \\
Total & 10,3 & 7,7 & 9,5 \\
N & 100 & 100 & 100 \\
& $(29)$ & $(13)$ & $(42)$ \\
\hline
\end{tabular}

Chi-cuadrado $=5,9217$

D.F. $=6$

$p=0,432$

Tabla Ib. Forma de conseguir empleo, \% por sexos.

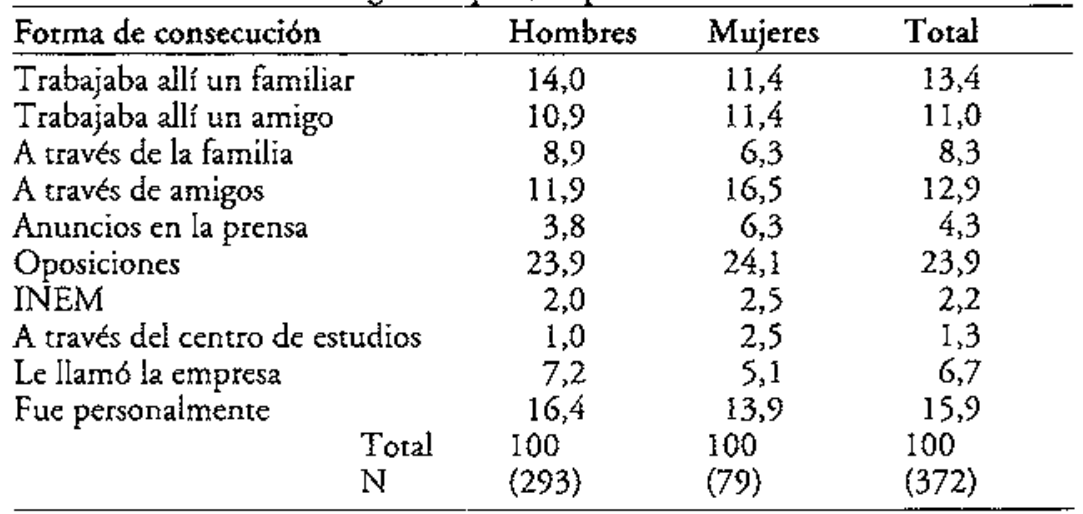

Chi-cuadrado $=4,5170$

D. F. $=9$

$\mathrm{P}=0,8742$ 
En la tabla 2 se observan las medias y las desviaciones estándar de los diferentes tipos de vínculos dentro de la red', así como las proporciones de cada uno de ellos y el tamaño de la red rotal según el género. Se aprecia que existen diferencias significativas entre la comparación de las redes personales de los hombres y de las mujeres.

La principal diferencia que se observa entre los sexos atendiendo a los diferentes valores de las medias es la variación en el tamaño de la red completa (6,48 para los hombres versus 6,00 para las mujeres), hecho que difiere con los resultados de los trabajos norteamericanos (Fischer, 1982; Moore, 1990), donde los valores son más similares.

En lo que respecta a las diferencias entre los tipos de vínculos, son los lazos familiares los más frecuentes en las mujeres, mientras que el resto de los vínculos aumentan en los hombres. Este hecho se suaviza cuando se observa la composición relativa de la red. La proporción de cada vínculo respecto a la red total es la siguiente: las mujeres se relacionan en mayor medida con individuos de todos los tipos de vínculos a excepción de los compañeros de trabajo. De lo cual se deduce que este componente es el que hace attmentar principalmente el volumen total de la red de los hombres. En términos absolutos, la diferencia entre el número de relaciones que integran las redes de los hombres y las de las mujeres consiste en que las redes masculinas disponen de menos vínculos familiares y más extrafamiliares, incluyendo más compañeros de trabajo que ningún otro tipo de relación. En números relativos, las mujeres comparadas con los hombres tienen redes con mayor número de familiares, vecinos, amigos y menor de compañeros de rrabajo.

De estos datos se sigue que los hombres de nuestra muestra tienen mayores oportunidades de interacción en el mundo laboral que las mujeres. Sin

Tabla 2. Diferencias de tamaño y composición de la red según el sexo.

\begin{tabular}{llll}
\hline Variables & $\begin{array}{l}\text { Varones } \\
\text { media } \pm \text { s.d. }\end{array}$ & $\begin{array}{l}\text { Mujeres } \\
\text { media } \pm \text { s.d. }\end{array}$ & $\begin{array}{l}\text { Significación } \\
\mathbf{p}^{*}\end{array}$ \\
\hline $\begin{array}{llll}\text { Red completa } \\
\text { Composición absoluta }\end{array}$ & $6,48 \pm 4,51$ & $6,00 \pm 3 ., 77$ & $<0,05$ \\
$\quad$ familiar & $1,99 \pm 1,53$ & $2,00 \pm 1,39$ & n.s. \\
extrafamiliar & $4,49 \pm 3,50$ & $4,00 \pm 2,89$ & $<0,01$ \\
$\quad{ }^{*}$ vecinos & $1,23 \pm 1,36$ & $1,19 \pm 1,22$ & n.s. \\
$\quad{ }^{*}$ amigos & $2,00 \pm 1,65$ & $1,99 \pm 1,58$ & n.s. \\
$\quad{ }^{*}$ compañeros de trabajo & $1,26 \pm 1,56$ & $0,81 \pm 1,21$ & $<0,01$ \\
Composición relativa (proporcion de...) & & \\
familia & $0,34 \pm .21$ & $0,36 \pm 0,19$ & n.s. \\
vecinos & $0,17 \pm .16$ & $0,19 \pm 0,17$ & n.s. \\
amigos & $0,30 \pm .18$ & $0,32 \pm 0,16$ & n.s. \\
compañeros de crabajo & $0,16 \pm .16$ & $0,11 \pm 0,15$ & $<0,01$ \\
\hline
\end{tabular}

Hombres respecto a mujeres

ni.s. = no tiene significación estadística 
embargo, parece que las mujeres tienen relaciones más diversas que los hombres, que prácticamente se centran en las del entorno laboral.

En la tabla 3 se muestran las correlaciones entre todas las variables consideradas y su significación según los sexos; los hombres por encima de la diagonal y las mujeres por debajo de la diagonal. En ella se aprecia que las variables reticulares y estructurales actúan de forma diferente en hombres y mujeres.

Del análisis minucioso de las correlaciones se pueden deducir los siguientes hechos. En primer lugar, se puede afirmar que hay diferencia sustancial entre las composiciones de las redes personales de los hombres y las mujeres en función de las posiciones diferenciales que unos y otros ocupan en la estrucrura social. En segundo lugar, se pone de manifiesto un funcionamiento peculiar en la composición y evolución de las redes de los hornbres y de las mujeres. Así, por ejemplo, conforme aumenta la edad, los hombres pierden un número menor de amigos que las mujeres; lo que se puede interpretar como que dicho aumento de edad implica determinadas obligaciones que limitarían la posibilidad de relación en las mujeres. Tal afirmación se confirma con el hecho de que las mujeres casadas presentan redes de menor rango que las que no lo

Tabla 3

\begin{tabular}{|c|c|c|c|c|c|c|c|c|c|c|c|c|c|c|c|c|c|}
\hline Variables & 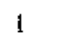 & 2 & 3 & 4 & 5 & 6 & 7 & 8 & 9 & 10 & 11 & 12 & 13 & 14 & 15 & 16 & 17 \\
\hline Edad & & & $.14^{*}$ & -.07 & -.07 & .02 & $.36 \%$ & -.06 & .09 & .03 & $.15^{\circ}$ & -.08 & .11 & .01 & $.18 \#$ & -.04 & -.14 \# \\
\hline $\begin{array}{l}\text { Educación } \\
\text { Empleo }\end{array}$ & & & $-.29 \#$ & $.46 \#$ & $.59 \%$ & .01 & .10 & .12 & $.16^{\circ}$ & .09 & .09 & .11 & $.16^{*}$ & -.03 &. $.17 \%$ & .05 & $.16^{\circ}$ \\
\hline jeuación laboral & -.02 & -.14 & & 288 & $-.39 \#$ & .08 & .05 & $.22 \#$ & -.11 & $-.24 \#$ & -.01 & $-.13^{\circ}$ & $-.38 \#$ & $.15^{\circ}$ & $.17 *$ & .10 & $-.67 \#$ \\
\hline Ocupación & -.22 & $.44^{\circ}$ & -.20 & & 444 & .05 & .05 & .01 & .03 & .02 &. $.15^{*}$ & .03 & .06 & $.0 \mathrm{i}$ &. $.15^{*}$ & .04 & .09 \\
\hline Ingresos & -.05 & $.46^{\circ}$ & -.31 & & & .03 & .02 & $-.17 \#$ & $.15^{x}$ & $.16^{*}$ & .01 & $.15=$ & $19 \#$ & .01 & $-.15^{*}$ & .01 & $.14^{*}$ \\
\hline rasado & .01 & .01 & -.09 & -.04 & .10 & & & .05 & .09 & .03 & .03 & .02 & .01 & .06 & .01 & -.05 & -.04 \\
\hline $\begin{array}{l}\text { Hijos } \\
\text { Red (absoluta) }\end{array}$ & .22 & .18 & .06 & -.11 & -.18 & .20 & & .06 & .05 & .05 & .10 & .04 & -.01 & .04 & .09 & -.04 & -.08 \\
\hline Complera & -.19 & .31 & -.28 & .23 & .39 & -.12 & -.13 & & & .964 & .63\# & $.78 \pi$ & .760 & $-.22 \#$ & .08 & .02 & .22 \\
\hline farniliat & -.03 & .14 & -.19 & .27 & .34 & -.05 & .01 & .64 & & & $.29 \%$ & 45 & $.45 \#$ & $.29 \%$ & $.14^{*}$ & $-19 \#$ & .03 \\
\hline Extrafamiliar & -.22 & .31 & .26 & .18 & .34 & -.13 & -.16 & $.96 \%$ & .39 & & & $.79 \#$ & $.79 \#$ & $-.42 \#$ & $.16^{\circ}$ & .07 & .30 \\
\hline Vecinos & .12 & .02 &. .01 & .10 & .16 & -.19 & .08 & $.72 \mathrm{~F}$ & .35 & $.73 *$ & & $.32 \#$ & $.32 \%$ & $-.32 \#$ & .684 & $-.20 \#$ & -.06 \\
\hline Amigos & $-.40^{*}$ & .39 &. .07 & .16 & .16 & -.15 & $-.2 !$ & $.79 \sharp$ & .22 & $.86 \%$ & $.51^{\circ}$ & & $.43 \%$ & $-.33 \#$ & -.11 & $.46 \#$ & .04 \\
\hline Comp. & -.15 & .31 & $-.52 \#$ & .14 & $.48^{\circ}$ & .03 & -.27 & .73 .5 & .36 & $.74 \%$ & .26 & & & & -.12 &. $.15^{\circ}$ & .66\% \\
\hline opcrion & .17 & -.13 & .17 & .12 & -.11 & .07 & .08 & -28 & .36 & $-.47^{x}$ & .38 & $-.44^{*}$ & $\cdot .28$ & & & & $0.4<t$ \\
\hline oporción vecinos & .32 & -.24 & .27 & -.22 & -.13 & -.23 & .18 & -.01 &. .21 & .07 & $.52 \mathrm{fi}$ & .01 & -.26 & $-.47^{\circ}$ & & & $-.27 \#$ \\
\hline soporción amigos & $-46^{\circ}$ & .25 & .01 & -.07 & -.08 & -.03 & .07 & .23 & -.29 & .39 & .18 & $.63 \mathrm{fH}$ & .02 & $-.70 \mathrm{H}$ & & & $.19 \#$ \\
\hline Prop. com. trabajo & -.18 & .23 & -.584 & .12 & $.40^{\circ}$ & .18 & -.15 & .22 & -.05 & .28 & -.18 & .08 & $.72 \pi$ & -.36 &. $.44^{*}$ & & \\
\hline
\end{tabular}

Hombres por encima de la diagonal y mujeres por debajo de la diagonal.

Nota: I = edad; 2 = educación; 3 = situación laboral; 4 = ocupación; 5 = ingresos; 6 = casado; 7 = hijos; $8=$ red completa; $9=$ red familiar; $10=$ red no familiat; $11=$ ted de vecinos; 12 = red de amigos; 13 = red de compañeros de trabajo; 14 = proporción de familia en la red; 15 = proporción de vecinos; 16 = proporción de amigos; 17 = proporción de compańeros de trabajo.

$\#=\mathrm{p}<.00$ !

$*=p<.01$ 
están, y de igual forma el incremento en el número de hijos también hace disminuir el rango de las redes personales de las mujeres.

Por otra parte, aunque, según se comprobó en la tabla l, las mujeres tienen en sus redes menos vínculos extrafarniliares que los hombres; sin embargo, cuando aumenta el grado de instrucción formal, las mujeres llegan a tener más individuos extrafamiliares en sus redes personales que los hombres (aunque la diferencia no sea muy grande). Lo mismo ocurre con el número de amigos. Aunque en términos generales aquéllas tienen menos amigos como miembros de sus redes personales, cuando la mujer aumenta su nivel educativo entonces tiene mayor número de amigos en la composición de sus redes personales que los hombres a igual nivel de instrucción. Y lo mismo podría decirse respecto a los compañeros de trabajo.

Efectivamente, la composición de las redes personales de las mujeres consta de un número menor de compañeros de trabajo que la de los hombres, sencillamente porque hay menos mujeres que ocupan posiciones en la población activa, pero cuando se hace un análisis diferenciado para el grupo de las mujeres y éstas ocupan posiciones laborales, entonces tienen mayor cantidad de compañeros de trabajo en la composición de sus redes que los hombres. Lo cual no quita para que, según nuestros datos, siga existiendo discriminación respecto a las mujeres, puesto que a igualdad de octupación los hombres perciben ingresos algo superiores. Por otra parte, el aumento de los ingresos hace a la mujer más interactiva con compañeros de trabajo que los hombres, y en general cuando se incrementan los ingresos, las mujeres tienen redes personales con rango mayor que los hombres.

La ocupación no significa, sin embargo, un impedimento para que las mujeres se relacionen también más que los hombres con familiares y parientes o con vecinos.

\section{Conclusiones}

De todo lo dicho anteriormente se sigue que las posiciones sociales diferenciales para el hombre y la mujer es un hecho determinante en la composición interna de sus respectivas redes sociales, así como que gran parte de las diferencias en la estructura reticular entre los géneros se ve afectada por la desigualdad estructural que incide sobre las rmujeres. Estas, al ocupar menos y peores posiciones laborales que los hombres, ven compuestas sus redes personales en mayor proporción por individuos pertenecientes a su entorno familiar.

Sin embargo ocurre, según se demuestra en los datos, que cuando la mujer alcanza posiciones laborales de competencia idéntica a las del hombre, entonces sus redes alteran bruscamente su composición sobrepasando con creces en número de compañeros de trabajo a los hombres.

Desde el punto de vista de la composición de sus redes sociales, las mujeres, cuando ih estructura les permite ocupar posiciones (principalmente laborales) similares a las de los hombres, no sólo tienen más compañeros de trabajo, sino también más familiares y vecinos. Es decir, las mujeres tienen redes socia- 
les menores y compuestas por más familiares porque tienen menos oportunidades de interacción en el mundo laboral, pero cuando éstas acceden a dicho entorno (en igualdad con el hombre) entonces sus redes son mayores y de composición más diversificada. La rnujer, en suma, cuando dispone de oportunidades de hacerlo, interacciona más que el hombre; lo que ocurre es que la estructura social copada por los hombres no deja hueco para que las mujeres ocupen las mismas posiciones.

\section{Bibliografía}

BLAU, P.M. (1977). Inequality and Heterogeneity: a Primitive Theory of Social Structure. Nueva York: Free Press.

Booth, A. (1972). "Sex and social participation". American Sociological Review, núm. 37, p. $183-192$.

BurT, R.S. (1983). "Range». En BurT, R.S. \& MinoR, M.J. eds., Applied Network Analysis: A Methodological Introduction, p. 176-194, Beverly Hills, CA: Sage.

Dęm, R. (1982). “Women, leisure and inequality». Leisure Studies, núm. 1, p. $229-246$.

FISCHER, C.S. (1982). To dwell among friends. Personal networks in town and city. Chicago: University of Chicago Press.

FISCHER, C.S. \& OLIKER, S.J. (1983). "A research note on friendship, gender and life cycle", Social Forces, núm. 62, p. 124-133.

GRANOVETTER, M.S. (1973). "The strenght of weak ties". American Joumal of Sociology, núm. 78, p. 1360-1380.

- (1974), Getting a job. A study of contacts and careers. Cambridge, Mass.; Harvard University Press.

Marsden, P.V. (1987). "Core Discussion Networks of Americans". American Sociological Review, núm. 52, p. 122-131.

Miller, J.B. (1976). Toward a New Psychology of Women. Boston: Beacon.

MoORE, G. (1990). "Structural Determinants of Men's and Women's Personal Networks". American Sociological Review, núm. 55, p. 726-735.

ReQuena, F. (1991). Redes sociales y mercado de trabajo. Madrid: CIS/Siglo XXI.

Wellman, B. (1985). «Domestic work, paid work and network». En DucK, S. \& PERLMAN, D. (ed.) Understanding personal relationships. Beverly Hills, California: SAGE, p. 159-192.

WINSTEAD, B.A. (1986), «Sex differences in same-sex friendships". En DerLEGA, V.J. $\&$ WINSTEAD, B.A. (ed.) Friendships and Social interaction. Nueva York: SpringerVerlag, p. 81-99. 